\title{
Thundercloud Induced Spatial Ion Flow in the Neighborhood of Rotating Wind Turbine and Impact Mechanism on Corona Inception
}

\author{
Wanshui Yu, Qingmin Li, Member, IEEE, Jiyao Zhao, Hongbo Li and Wah Hoon Siew, Senior \\ Member, IEEE
}

\begin{abstract}
Lightning accidents seriously threaten safe operation of the wind turbines due to unclear initiation mechanism of the airborne lightning induced discharges on turbine blades, in which turbine rotation is one of the principal influencing factors. To study the impact mechanism of wind turbine rotation on corona discharge inception, a numerical model with dynamic meshing of charged ions in the neighboring space of large-scale rotating wind turbine during thunderstorm was established in this paper, and the validity of the model was verified by long gap discharge experiments on a scaled wind turbine. Based on the proposed model, the spatial and temporal distribution of charged particles in the neighboring area of the rotating wind turbine as well as the space charge-caused local electric field distortion scenario were obtained. The influence mechanism of blade rotation on corona discharge inception was further analyzed and elucidated accordingly. The results indicate that, the charged particles are unevenly distributed near the rotating blade tip, and the contours present a strip-like shape, the critical area of which may facilitate corona discharge inception. As the blade speed increases from 6rpm to 20rpm, the E-field extremum at the blade tip increases by $38 \%$, causing the blade tip prone to initiate corona discharge. The critical rotating speeds corresponding to corona inception probability were calculated under different thunderclouds-determined field strengths and a safe boundary was defined, by which it is recommended that wind turbines operate at a reduced speed below $8 \mathrm{rpm}$ under thunderclouds conditions.
\end{abstract}

Index Terms-Wind turbine, rotating blade, ion flow distribution, charge density descent vector, corona discharge

\section{INTRODUCTION}

I $\mathrm{N}$ recent years, wind power generation has developed on a large scale worldwide. According to the statistical data from Global Wind Energy Council (GWEC), the new installations surpassed 90GW in 2020, among which China's new wind power capacity accounted for $56 \%$; at the end of 2020 , the global cumulative wind power capacity was up to $707 \mathrm{GW}$, and

This work was supported by the National Natural Science Foundation of China (Grant No. 51929701, 51737005 and 51420105011), the Beijing Natural Science Foundation (Grant No. 3202031). (Corresponding author: Qingmin Li)

Wanshui Yu, Qingmin Li, Jiyao Zhao and Hongbo Li are with the State Key Laboratory of Alternate Electrical Power System with Renewable Energy Sources (North China Electric Power University), Beijing 102206, China (e-mail: lqmeee@ncepu.edu.cn).

Wah Hoon Siew is with the Department of Electronic \& Electrical Engineering, the University of Strathclyde, Glasgow G1 1XQ, UK.
China accounted for $40 \%$. With the increase of the single unit capacity of wind turbines, the tower and the diameter of the blades increase as well, and the wind turbines become more susceptible to lightning strikes. The lightning damage rate of wind farms in Texas, Kansas and Illinois in the United States was 11.9 units/ (100 units• year) [1] from 2009 to 2013 on the basis of the statistics; and it was as high as 9.8 units/ (100 units• year) [2] among 132 wind turbines at four wind farms in Yunnan Province, Guizhou Province and Shanxi Province from 2012 to 2017 . Wind turbines are valuable equipment in wind farms, whose cost takes up more than sixty percent of the investment in wind power projects. Once the wind turbines get struck, the huge power released from the lightning might cause severe damages to the wind turbines, and even make them shut down, causing great economics losses.

The study on lightning strike process of wind turbine is necessary to improve the lightning protection capabilities for wind turbines. As early as the 1990s, Nordic countries have launched research on the wind turbine lightning protection. Danish Ministry of Energy Research and Development released a piece of suggestions for lightning protection of wind turbines [3] in 1999, based on which IEC successively published multiple versions of wind turbine lightning protection standards[4], [5]. The main idea of wind turbine lightning protection is to install metal conductors to the blades and then lead the lightning current to the ground. Different kinds of lightning protection systems for wind turbine blades recommended by IEC are shown in Fig 1.
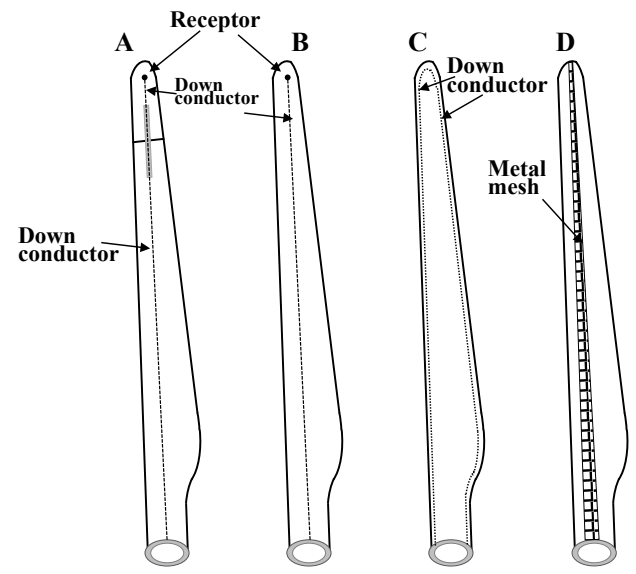

Fig 1. Lightning protection systems for wind turbine blades recommended by IEC 
Even if complete lightning attachment system has been installed on the blade during the manufacturing process, the lightning protection for wind turbines is still insufficient with frequent lighting accidents. This is because the attachment system mainly aims at the stationary wind turbines, weakly taking the rotating effect of blade into consideration.

While according to the observation data from wind farms, the blades were struck by lightning mostly in a rotating state [6], and the rotation effect aggravated the damage to the blades caused by lightning strikes. On the basis of the three-dimensional lightning observation array and the lightning observation data of wind farms, $\mathrm{J}$ Montanyà [7] proposed that rotating wind turbines are more likely to initiate upward leaders. M Ishii [8] observed twelve wind turbines for five years in a wind farm along the coast of Japan and found that rotating blades are more susceptible to lightning strikes than stationary blades when the blade angle exceeds $30^{\circ}$. The influence of blade rotation on the lightning discharge was discovered initially from wind farm observation, from which the impact mechanism remains to be investigated.

To further study the physical characteristics of lightning strikes on rotating wind turbine, scholars have conducted extensive experimental studies on wind turbine. B M Radičević [9] carried out a 3-meter gap discharge experiment on a 1:40 scaled wind turbine. It was found that fewer strikes hit the blade receptors while the number of discharges from the electrode to ground increased in the rotating condition compared to the stationary condition. However, the experimental results are inconsistent with the observation results of natural lightning in the wind farms. B M Radičević explained that due to the rotation of the blades, the positive space charges could not concentrate near the blade tip with higher rotating speed, therefore the field strength in the air gap was reduced, making the breakdown more difficult. Wang Yu [10] et al. conducted discharge experiment of scaled wind turbine under different gap lengths, and found that the breakdown voltage reduced when rotating speed increased in a lager air gap $(>4 \mathrm{~m})$, which was in accordance with actual lightning observations. The space charge may have different effects under different lengths of air gaps. In short gaps, it is more inclined to affect the breakdown voltage of the gap. In the experiments, it is possible to generalize the conclusions from the discharge phenomena, without deeply studying the effect of space charge.

Furthermore, since the long gap discharge experiment of scaled wind turbine still cannot entirely reproduce the lightning process of rotating wind turbines in wind farms, the scholars carried out simulation work for the lightning strike process of the wind turbine. $\mathrm{M}$ Alonso [11] et al. established the three-dimensional model of the wind turbine, and the background electric field by using Malan's three-layer thundercloud structure model was calculated. They found that the electric field strength is about $75 \%$ higher than that in the stationary state, in which way it was believed that the blades under rotation state are more susceptible to lightning strikes. Montanyà [7] qualitatively explained the space charge distribution near the tip of the rotating wind turbine and believed that the space charge near the tip was drawn into layers distribution due to the blade rotation. Qu [12] et al. established a calculation model for the space charge distribution near a single receptor of wind turbine blade under the effect of the thundercloud and analyzed the influence of rotating speed on charge distribution. It was proposed that the higher the wind speed, the greater the corona current on the surface of the receptor. However, the rotation effect was not taken into consideration. In the lightning protection on grounding structures [13]-[15] and transmission lines [16], [17], intensive researches related to ions distribution under thundercloud have been carried out. But for wind turbines, the rotation factor increases the difficulty of the numerical calculation. Since the blade rotation is a critical factor that influences the drift and diffusion of charged ions near blade tip, which will further affect the corona inception, it is necessary to carry out research of charged ions distribution near the rotating wind turbine and the impact mechanism on corona inception.

In this paper, a convection and diffusion model of thundercloud induced ions flow near rotating wind turbine was established by employing rotational meshing methodology, according to which the impact mechanism of blade rotation on corona inception was further studied. The relationship between rotating speed and blade position angle was found to be a U-shaped curve, which can be used to determine the critical speed boundary of wind turbine corresponding to corona inception under thundercloud conditions.

\section{CONVECTION AND DIFFUSION MODEL OF CHARGED PARTICLES IN THE NEIGHBORING SPACE OF A ROTATING WIND TURBINE}

\section{A. Establishment of the rotating ions flow model}

A 2-D convection and diffusion model of ions flow was established in this paper and the model diagram is shown in Fig. 2.

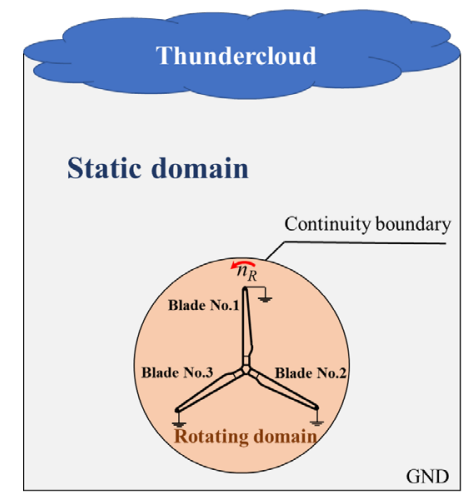

Fig. 2. Computational modeling diagram

Based on the atmospheric observation and measurement result, the electric field strength of the thundercloud was at $20 \sim 60 \mathrm{kV} / \mathrm{m}$. Furthermore, since $90 \%$ of the lightning in nature are negative in polarity, the electric field strength of the thundercloud was set as $-40 \mathrm{kV} / \mathrm{m}$, while the blade tips were grounded. The wind turbine model was established according to the geometric dimensions of the actual operating wind turbine. Because the blade tip is made of metal, the rotating impeller (with a rotating speed of $n_{\mathrm{R}}$ ) has a shielding effect on 
the wind turbine tower in a thunderstorm environment, so that the effect of the tower was ignored in the modeling process. According to the size of a typical $2 \mathrm{MW}$ wind turbine, the height of the wind turbine hub was set to $80 \mathrm{~m}$ from the ground and the blade length was $50 \mathrm{~m}$ in this paper. The calculation solution boundary should be much larger than the size of the wind turbine model to avoid the influence of the scale effect. In this article, the calculation solution area was set to a square with a side length of $500 \mathrm{~m}$.

There exist mainly aerosol particles, nitrogen molecule and oxygen molecule in the air. When the gas molecules in air are ionized, small ions like nitrogen ions, oxygen ions will be generated. The charged aerosol particles will be generated by the recombination of the small ions with aerosol particles. Under the thundercloud background electric field, the ionization of gas molecules will occur near the blade tip because that the metal tip is grounded through the down conductor. Since $90 \%$ of the lightning in nature are negative in polarity, it is positive charged particles that mainly distribute near the blade tip under the influence of negative thundercloud electric field. So negative charged particles are neglected during the discharge process. In that way, the particles near the blade tip were simplified into three types, namely small positive ions, positive charged aerosol particles and neutral aerosol particles. The discharge process can be simplified into interaction among these three kinds of particles, described by the following charged particle convection/diffusion equations:

$$
\begin{aligned}
& \frac{\partial n_{+}}{\partial t}=D \cdot \nabla^{2} n_{+}-\nabla \cdot\left(n_{+} \cdot \mu_{n_{+}} \cdot \boldsymbol{E}\right)-k_{n_{+} N_{0}} \cdot n_{+} \cdot N_{0} \\
& \frac{\partial N_{+}}{\partial t}=D \cdot \nabla^{2} N_{+}-\nabla \cdot\left(N_{+} \cdot \mu_{N_{+}} \cdot \boldsymbol{E}\right)+k_{n_{+} N_{0}} \cdot n_{+} \cdot N_{0} \\
& \frac{\partial N_{0}}{\partial t}=D \cdot \nabla^{2} N_{0}-k_{n_{+} N_{0}} \cdot n_{+} \cdot N_{0}
\end{aligned}
$$

where $n_{+}$is small positive ions, $N_{+}$is positive aerosol particles, $N_{0}$ is neutral aerosol particles in the atmosphere, $\mu$ is the mobility of the charged particles, with values of $\mu_{n_{+}}=1.5 \times 10^{-4} \mathrm{~m}^{2} \cdot \mathrm{s}^{-1} \cdot \mathrm{V}^{-1}$ for small positive ions, $\mu_{N_{+}}=1.5 \times 10^{-6} \mathrm{~m}^{2} \cdot \mathrm{s}^{-1} \cdot \mathrm{V}^{-1}$ for positive aerosol particles. The attachment coefficient of the small positive ions to neutral aerosol particles $k_{n_{1} N_{0}}$ is equal to $2.9 \times 10^{-12} \mathrm{~m}^{3} \cdot \mathrm{s}^{-1}$. The value of diffusion coefficient $D$ is $1 \mathrm{~m}^{2} / \mathrm{s}$. The selection of the above parameters comes from the related research of atmospheric electricity [18]. $\boldsymbol{E}$ is the electric field strength in space, coupled with the physical field of point particle drift and diffusion, calculated by the Poisson equation:

$$
\nabla \cdot \boldsymbol{E}=-\nabla^{2} \Phi=\frac{e \cdot\left(n_{+}+N_{+}\right)}{\varepsilon_{0}}
$$

where $\Phi$ is space potential, $e$ is elementary charge, $\varepsilon_{0}$ is vacuum dielectric constant.

Before the thundercloud electric field formed, it is assumed that no ionization and other processes of charged particles occurred, so the air is considered being electrically neutral. The initial density of neutral aerosol particles differs according to different atmosphere conditions, which is about $5 \times 10^{9} / \mathrm{m}^{3}$ in areas with good air conditions while exceeding $1 \times 10^{11} / \mathrm{m}^{3}$ in urban area or dirty-polluted area. In this article, the initial value of $N_{0}$ was set to $10^{11} / \mathrm{m}^{3}$ [19], and the metal parts of the blade tip were set to ground. In addition, the initial values of small positive ions $n_{+}$and positively charged aerosol particles $N_{+}$ were set to 0 . However, with no potential source terms equation (1) will provide any spatiotemporal distribution of small positive ions and positively charged aerosol particle, so the value was taken as $10^{-6} / \mathrm{m}^{3}$ in actual calculating process which was tiny enough compared with the initial value of $N_{0}$.

To solve the rotating calculation in this model, the calculation domain is newly meshed in each time step under traditional considerations, whereas the calculation is less efficient. In this paper, dynamic mesh was employed to the calculation domain. The area occupied by the impeller and the area outside of impeller were set as rotating and static computing domain respectively, which were meshed independently. And the boundary of the two domains was set as continuity boundary condition in order to make the continuous transition of numerical calculation. Given that the center of the rotation domain circle is $\left(x_{0}, y_{0}\right)$, the coordinates of the rotation mesh cell at moment $t$ are $\left(x_{t}, y_{t}\right)$, and the coordinates of the mesh cell at moment $t+1$ are $\left(x_{t+1}, y_{t+1}\right)$ after a time step $\Delta t$, then the rotation process of mesh cell is shown as follows:

$$
\left[\begin{array}{c}
x_{t+1} \\
y_{t+1}
\end{array}\right]=\left[\begin{array}{cc}
\cos \left(\frac{\pi n_{R}}{30} t\right) & -\sin \left(\frac{\pi n_{R}}{30} t\right) \\
\sin \left(\frac{\pi n_{R}}{30} t\right) & \cos \left(\frac{\pi n_{R}}{30} t\right)
\end{array}\right] \cdot\left[\begin{array}{c}
x_{t}-x_{0} \\
y_{t}-y_{0}
\end{array}\right]+\left[\begin{array}{c}
x_{0} \\
y_{0}
\end{array}\right]
$$

where $n_{\mathrm{R}}$ represents to the rotating speed of wind turbine, depending on the actual operating wind turbine speed, taking 6 20 rpm.

In the rotating domain, since the cells of the computation mesh are rotating, the formula for solving positive ion $n_{+}$in equation (1), for example, has the following expression:

$$
\left\{\begin{array}{l}
\lim _{\Delta d=0} \frac{n_{+}\left(x_{t+1}, y_{t+1}\right)-n_{+}\left(x_{t}, y_{t}\right)}{\Delta d}=D \cdot \nabla^{2} n_{+}\left(x_{t}, y_{t}\right)- \\
\left.\nabla \cdot\left(n_{+}\left(x_{t}, y_{t}\right) \cdot \mu_{n_{+}} \cdot \boldsymbol{E}\right)-k_{n_{+} N_{0}} \cdot n_{+}\left(x_{t}, y_{t}\right) \cdot N_{0}\left(x_{t}, y_{t}\right)\right) \\
\Delta d=\sqrt{\left(x_{t+1}-x_{t}\right)^{2}+\left(y_{t+1}-y_{t}\right)^{2}}
\end{array}\right.
$$

To solve the convection and diffusion equations of charged ions, a commercial finite element method software COMSOL Multiphysics was employed in this paper. The calculation domain was divided into sub-area meshes to reduce the computing cost while ensuring the computational reliability. In the rotation domain, extreme fine cells (from the size order of $10^{-4} \mathrm{~m}$ to $10^{-1} \mathrm{~m}$ ) were structured in the meshing process near the blade tip. While the static domain was set as larger mesh (from the size order of $10^{-1} \mathrm{~m}$ to $10^{1} \mathrm{~m}$ ) structure. In the meantime, the meshes on the boundaries of the rotation and static domain were set as the same size to avoid the circumstance of numerical discontinuity on the rotation boundary. 


\section{B. Validation of the rotating ions flow model}

Long gap discharge experiment was conducted on a scaled wind turbine to verify the validation of the model, whose setup is shown in Fig. 3. The scaled wind turbine is a 1:30 reduced model of a typical $2 \mathrm{MW}$ wind turbine. The blade was 1.8 meters long and the receptor of the blade tip was grounded through the tower and the down conductor. The rod electrode was 2 meters higher from the blade tip which was connected to the impulse voltage generator.

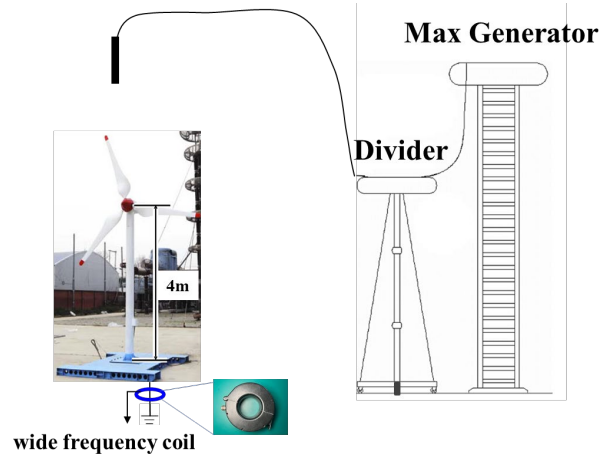

Fig. 3. Long-gap discharge experiment platform of scaled wind turbine

By applying negative switching impulse voltage of $250 / 2500 \mu$ s with different peak values (lower than breakdown voltage) on the rod electrode, the transient current in the grounding line was measured to characterize the magnitude of the charge generated by the blade tip during pre-discharges. The frequency response of the current sensor ranges from $10 \mathrm{kHz}$ to $30 \mathrm{MHz}$ and the measurable current peak is $400 \mathrm{~A}$.

The calculation area of the simulation was reduced to $20 \mathrm{~m} \times 8 \mathrm{~m}$ to keep consistent with the experiment. Because the discharge near the blade tip mainly occurs at the rise edge of the operation impulse voltage, the time range is set to $400 \mu$ s in calculation, and the 0 to $400 \mu$ s part of the standard switching impulse voltage was applied to the rod electrode in the model. The peak values of applied impulse voltage in the experiments were set as $-500 \mathrm{kV},-600 \mathrm{kV},-700 \mathrm{kV}$ and $-800 \mathrm{kV}$. A case of current measurement with $-600 \mathrm{kV}$ switching impulse voltage is shown in Fig. 4.

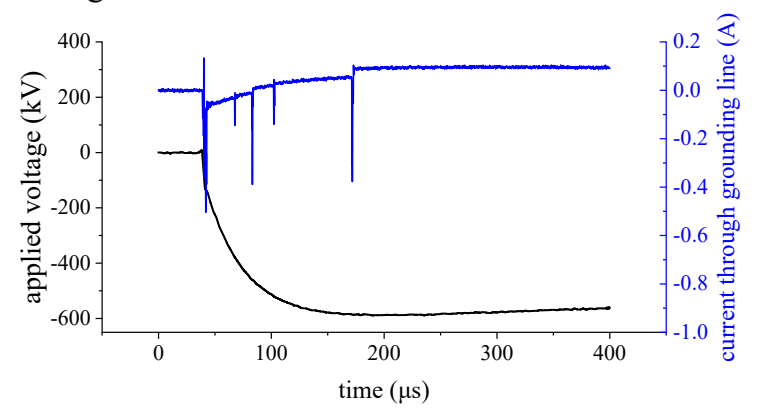

Fig. 4 A case of the applied voltage and measured current

The current waveform contains displacement current caused by the geometry capacitance between electrode and blade tip. The displacement current is calculated as:

$$
i_{g m}(t)=C_{g m} \times \frac{d U_{a p p}(t)}{d t}
$$

where $C_{\mathrm{gm}}$ is geometry capacitance, $\mathrm{F} ; U_{\mathrm{app}}(t)$ is the applied voltage on electrode, $\mathrm{V}$.

$U_{\text {app }}(t)$ is easy to obtain according to the voltage divider. The geometry capacitance was calculated to be $11.9 \mathrm{pF}$ with setting equivalent geometric model of the experimental platform in Comsol. By subtracting the displacement current from the original current waveform, the current generated by the pre-discharge activity at the blade tip is obtained.

In the simulation model, the total charge corresponding to the charged ions accumulated near the blade tip was calculated in comparison with the integral value of the experimentally measured current in the grounding line at each voltage condition. The comparison is shown as Fig. 5.

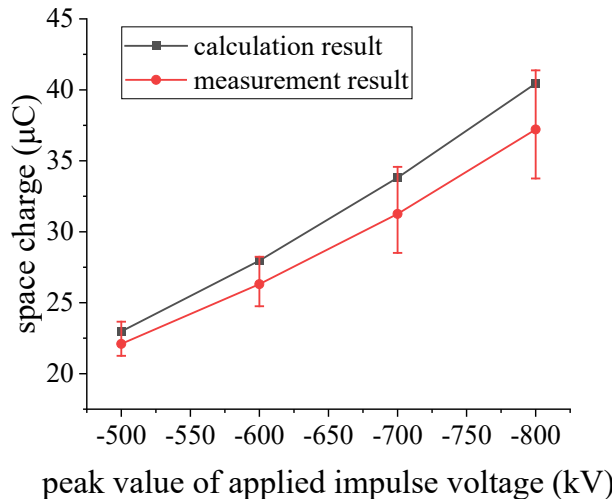

Fig. 5. Comparison between simulation and experimental results

As seen in Fig. 5, the results of the simulation calculation are in good agreement with the experimental measurements, and there is a consistent trend in the amount of charge obtained as the applied switching voltage increases. However, the calculated charge is slightly larger than the experimental measurement. This is because the charge near the blade tip measured in the experiment was determined by the integral of measured current. However, not all the charges near the blade tip flowed into the tip and passed through the ground, so the measured experimental results were slightly smaller than the simulation calculations.

\section{DISTRIBUTION CHARACTERISTICS OF CHARGED PARTICLES IN THE NEIGHBORING SPACE OF ROTATING WIND TURBINE}

Under the influence of negative polarity thundercloud electric field, positive charged particles were generated near the wind blade tip. The distribution of positive charged particles (the sum of small positive particles and positive aerosol particles) was calculated at the rotation speed of $12 \mathrm{rpm}$.

The positive ions distribution at the beginning of the blade rotation (rotation angle $1^{\circ}$ ) is shown in Fig. 6. As the blade just began to rotate, the space charge near the blade tip presents an approximately symmetrical distribution. We define the region where the density of charged particles is greater than $10^{12}$ as the charge-dense region. There exists a circle-like charge-dense region in the neighboring area of blade tip. The space charge 
decays rapidly towards both vertical and horizontal directions, and decay from $10^{14}$ to $10^{10}$ within a few meters.

Under the combined influence of the electric field of thundercloud and the rotation effect of blade, charged particles will migrate under the influence of electric field on the one hand, and form asymmetric distribution under the influence of blade rotation on the other hand. Therefore, the distribution of charged particles has a large change during the rotation of wind turbine. The distribution of charged particles under rotation angle of 30 degree is as shown in Fig. 7. The contour map of positive ions is dragged from quasi-circular to quasi-elliptical. The fraction of positive ions in the direction of blade rotation decays rapidly, while the tangential fraction in the opposite direction of blade rotation decays slowly. And the charge-dense region shows a "strip-like" distribution rather than a "circle-like" distribution.

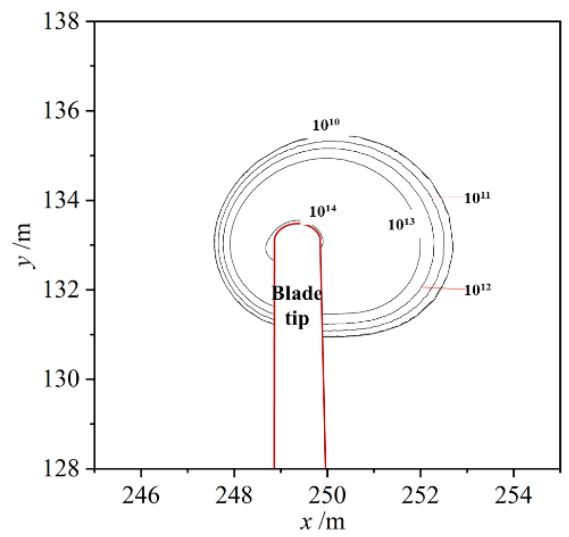

Fig. 6. Contour of positive ions density distribution at the start of blade rotation $\left(1 / \mathrm{m}^{3}\right)$

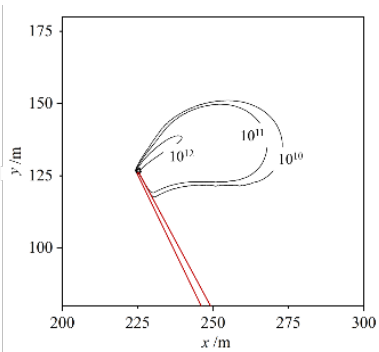

(a)

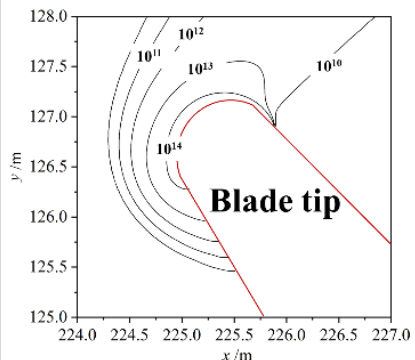

(b)
Fig. 7. Contour of positive ions density distribution when blade rotates to $30^{\circ}$ $\left(1 / \mathrm{m}^{3}\right)($ a) counter of positive ions, (b) enlarged view.

And in the literature [7], a qualitative analysis of the distribution of space charge near the blade tip in the case of rotation was conducted. It was analyzed that, due to the rotation, the total corona charge $q_{\mathrm{c}}$ is distributed in layers with density $\rho$. In such a case the charge amount just above the tip at any position is much lower than in the stationary case, as shown in Fig. 8. Since the charge injected into the air drifts with the electric field, it will form radially periodic features separated by a distance $d$ related to the rotation speed and the ion drift.

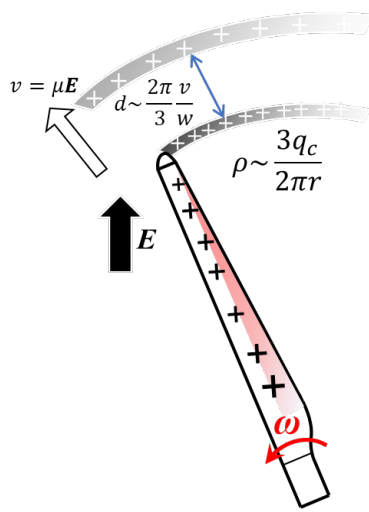

Fig. 8. Qualitative analysis of blade rotation on ions distribution in literature [7]

The stripe-like distribution of ions distribution (in Fig. 7) calculated by the proposed model in this paper is consistent with the proposed layer-shape distribution in Fig. 8. However, the charge distribution didn't show separation feature depicted by literature [7]. The reason is that the charge density should be continuous according to the continuity equations.

Affected by space charge, the space potential near the rotating blade also changes, as shown by the arrow in Fig. 9 . Positive ions accumulate on the blade side opposite to the rotating direction, as shown in Fig. 7(a), thus causing a positive space potential area on this side in Fig. 9(b). The ionization and recombination of charged particles will be more frequent in this area.

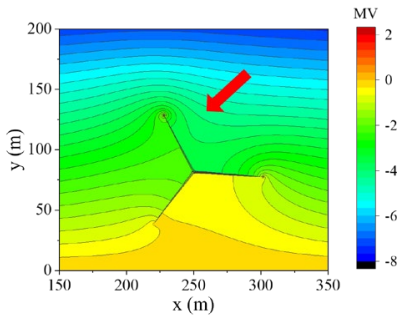

(a)

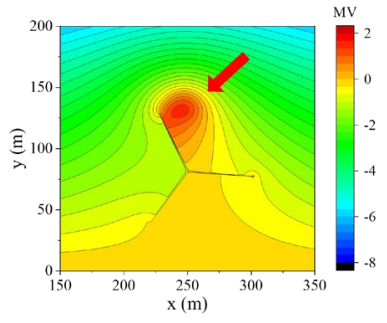

(b)
Fig. 9. Space potential contour near blade tip under the influence of space charge (a) without space charge, (b) with space charge.

Due to the influence caused by blade rotation, the density distribution of in different directions near the blade tip is also more uneven. In order to analyze the difference of ions distributions on different directions of blade tip, and further analyze its influence on the corona discharge, the intercept lines of the blade tip in three different directions were taken as shown in Fig. 10(a) (namely, the tangent line in the opposite direction of the blade tip rotation (intercept line 1), the intercept line in the vertical upward direction of the blade tip (intercept line 2) and the horizontal intercept line in the direction of the blade tip rotation (intercept line 3)). The results of the positive ions distribution on the three intercept lines during the blade rotation are shown in Fig. 10(b). 


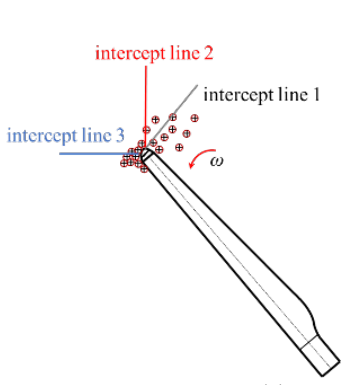

(a)

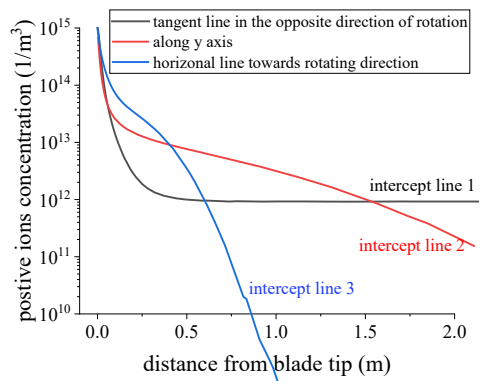

(b)
Fig. 10. Density distribution of positive ions on the intercept lines of blade tip (a) schematic diagram of intercept lines from blade tip, (b) density distribution of positive ions on the intercept lines at an angle of $30^{\circ}$.

From Fig. 10(b), it can be seen that on the intercept line 3, namely along the horizonal line towards rotating direction, more charged particles (ranging from $10^{15}$ to $10^{13} / \mathrm{m}^{3}$ ) accumulate near the blade tip within $0.5 \mathrm{~m}$, while the charged particles density decreases rapidly further away from the blade tip ( $0.5 \mathrm{~m}$ away); along the direction of intercept line 1 , fewer charged particles (ranging from $8 \times 10^{14}$ to $10^{12} / \mathrm{m}^{3}$ ) accumulate near the blade within $0.5 \mathrm{~m}$ and are more uniformly distributed far away from the blade tip; while along the direction of intercept line 2 , the level of charged particles density near the blade is in between the results along intercept line 1 and intercept line 3. The accumulation of space charge has a distorting effect on the electric field near the blade tip. Due to the difference of charged particles density distribution on different directions of the blade tip, the space electric field varies a lot on different directions, and the results are shown in Fig. 11.

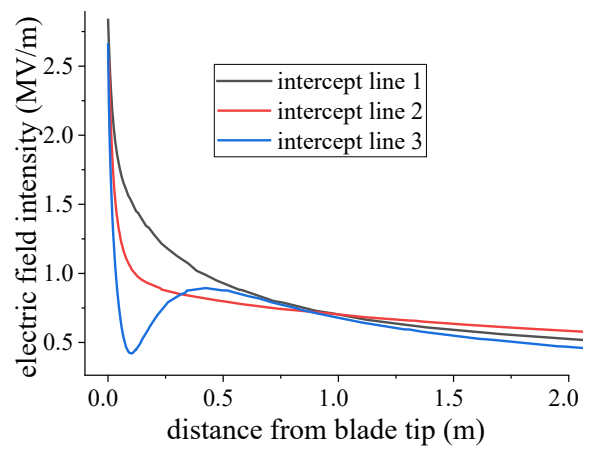

Fig. 11. Electric field curves on different directions from blade tip under the distortion effect of space charge

Along the direction of intercept line 1, the corresponding density of charged particles was the fewest within $0.5 \mathrm{~m}$ from blade tip. The distortion degree of space charge on electric field is the smallest, so the field strength in this area is higher than that on other two directions, making it easier to form corona discharge.

Along the direction of intercept line 3, more charged particles accumulate near blade tip, so the electric field distortion degree is greater, and it is more difficult to reach the initiation filed strength of corona discharge than that on intercept line 1 . In addition, along intercept line 2, the electric field strength near the blade tip is between the other two directions.

It can be concluded that due to the nonuniform distribution of charged particles near blade tip caused by rotation, there exist obvious differences among electric fields in different directions from blade tip. Discharging phenomenon is prone to incept along the tangent line in the opposite direction of rotation (i.e., on intercept line 1).

Similar phenomena were discovered in the simulated lightning attachment experiment of scaled wind turbine carried out by the authors of this paper, as shown in Fig. 12. A 1:30 scaled model of typical $2 \mathrm{MW}$ wind turbine was adopted in this experiment, in which the discharge gap was $2 \mathrm{~m}$. High-speed camera was used to capture the leader development and attachment process of rotating wind turbine.

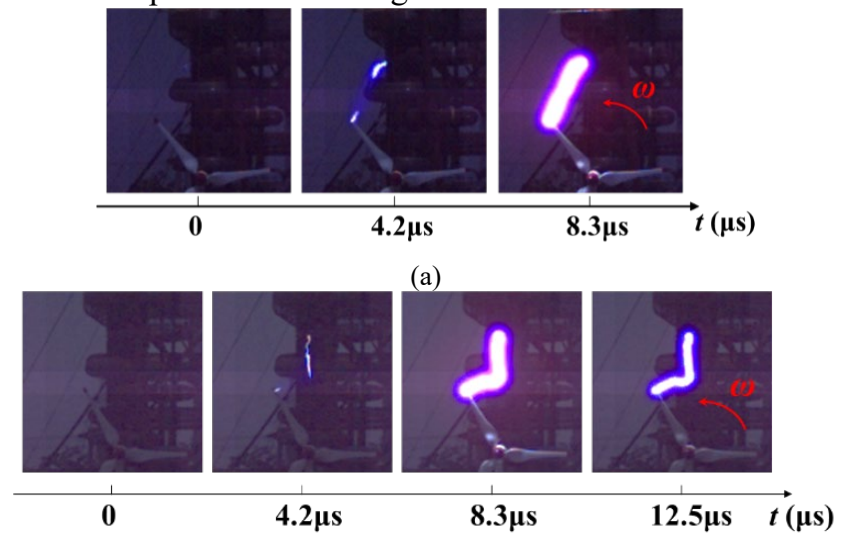

(b)

Fig. 12. Discharge paths of scaled wind turbine captured by high-speed camera (a) discharge example 1, (b) discharge example 2.

As can be seen from Fig. 12, in both results, discharge on the blade tip is formed on the side of the opposite direction of rotation (see the image of $\mathrm{t}=4.2 \mu \mathrm{s}$ ), which is then connecting to the downward leader and finally causing an attachment. It is noteworthy that in the second group of attachment, the discharge initiated from blade tip is formed along the tangent line in the opposite direction of blade rotation, the obtained results are in accordance with the analysis above.

\section{IMPACT MECHANISM OF BLADE ROTATION ON CORONA INCEPTION}

\section{A. Rotation effect on the electric field distribution in the neighboring space of blade}

The distribution of spatial ion flow near the blade tip under the influence of rotation was analyzed which shows agreement with the observation and experiment phenomenon. And the influence mechanism of blade rotation on corona inception near blade tip was further studied. On one hand, the field strength on the surface of the blade tip changes due to the change of its spatial position under the background field. On the other hand, the distribution of charged particles near the blade tip is also changing due to their drift under the influence of electric field and their own diffusion.

On the condition without the influence of space charge, the electric field strength at the blade tip shows a periodic change with the rotation angle (the angle $\theta$ between the blade and the vertical direction is defined as the rotation angle, shown in Fig. 13(a)). For a single blade, the electric field strength is the largest when it is in the vertical upward state $\left(0^{\circ}\right)$ and the 
smallest when it is in the vertical downward state $\left(180^{\circ}\right)$. Whereas the three blades have field shielding effect with each other, the electric field strength on the blade tip is the largest when blade rotates from -60 to 60 degree with the vertical direction, at this time the blade No. 2 and No. 3 are shielded by blade No.1. Similarly, when the blade No. 2 rotates from -60 to 60 degree with the vertical direction, blade No. 1 and No. 3 are shielded. Fig. 13(b) shows the electric field strength curve of each blade tip during the rotation of the wind turbine without the influence of space charge. The solid line shows the actual effective electric field strength of the blade tip after taking the shielding effect among blades into account.

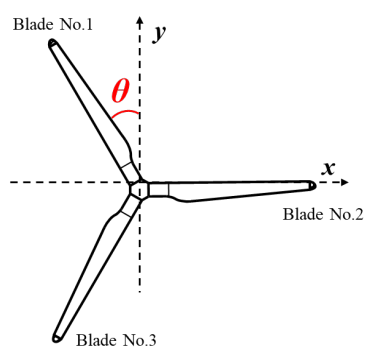

(a)

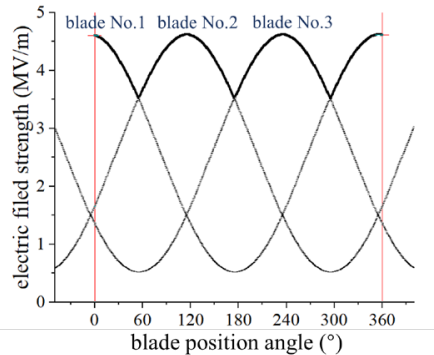

(b)
Fig. 13. Periodic variation of electric field strength on the blade tip of rotating wind turbine (a) the definition of blades and rotation angle (b) periodic variation of field strength

Since the variation of the electric field on the blade tip of 3 blades with rotation angle is periodic and symmetric, considering the computational cost, the rotation process of blades from rotation angle of 0 to 60 degrees was taken into consideration.

The distribution of charged particles near the blade tip during blade rotation is calculated, as shown in Fig. 14. At the beginning of the blade rotation (before 0.1s), the drift and diffusion of charged particles near the blade tip has not been fully developed. In the range of $2 \mathrm{~m}$ from the blade tip, the charged particles density is in the order of $10^{13}$, the electric field strength of the tip surface in this period is mainly affected by the distribution of charged particles density. The charged particles near the blade tip have moved to a farther area after $0.1 \mathrm{~s}$ and their accumulation effect near the blade tip is decreased. At this point, the charged particles density at $2 \mathrm{~m}$ from the tip reduced from $5 \times 10^{12} / \mathrm{m}^{3}$ to $10^{11} / \mathrm{m}^{3}$ from $0.1 \mathrm{~s}$ to $0.5 \mathrm{~s}$. Since the charged particles density has decayed to a lower level, the electric field strength on the surface of blade tip in this period is mainly influenced by the spatial position of the blade tip (i.e., the distance from thundercloud).

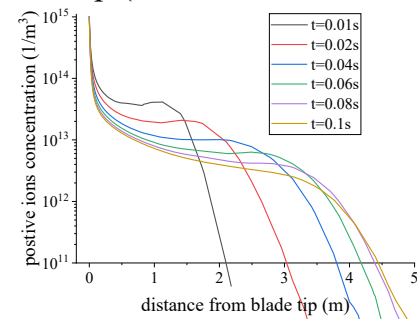

(a) (b)

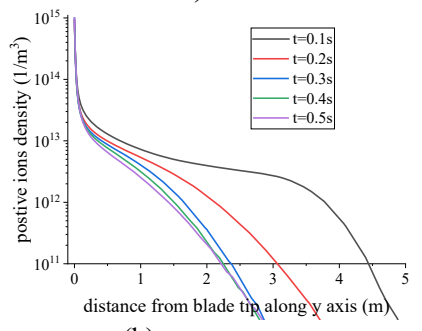

Fig. 14. Charged particles density near blade tip of rotating wind turbine (a) in the period of slight rotation stage (b) at the development stage of rotation.
Based on the analyzed of temporal distribution charged ions near the blade tip while rotating, the variation of the electric field strength on the blade tip with the rotation angle at different rotating speeds incorporating the effect of space charge was calculated as shown in Fig. 15.

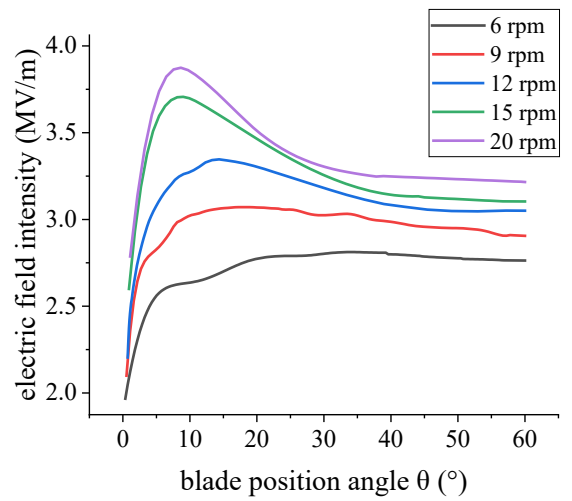

Fig. 15. Curve of electric field strength of blade tip with rotation angle at different speed

Corona discharge is considered to initiate near the blade tip when the electric field strength at the tip surface reaches the corona initiation field strength. The electric field strength for corona initiation is usually calculated by an empirical formula, namely the Peek formula[20]:

$$
\boldsymbol{E}_{\mathrm{cor}}=\boldsymbol{E}_{0} \cdot \delta \cdot\left(1+\frac{c_{1}}{\sqrt{r_{\text {tip }}}}\right)
$$

where $r_{\text {tip }}$ is the curvature radius of blade tip, with the value of $0.1 \mathrm{~m}$ in this study; $\delta$ represents the relative density of air with the value of $1 ; \boldsymbol{E}_{0}$ and $c_{1}$ are empirical constants of $2.7 \times 10^{6} \mathrm{~V} / \mathrm{m}$ and $0.054 \mathrm{~m}^{0.5}$, respectively. From equation (6), it is calculated that the initiation field strength of the corona discharge on the blade tip surface of this paper is $3.16 \mathrm{MV} / \mathrm{m}$.

As can be seen from Fig. 15, the general trend of the electric field strength on the blade tip at different rotating speeds is shown to increase and then decrease with increasing rotation angle. Combined with Fig. 14, it can be obtained that at the beginning of rotation, when the rotation angle is relatively small, the migration of space charge and its own diffusion is not sufficient, so the concentration of charged particles is formed in the vicinity of the blade tip. In this period the electric field strength of the blade surface is mainly affected by the space charge. With the migration and diffusion of space charge to the remote area of the blade tip, its weakening effect on the electric field near the blade tip gradually decreases, which shows that the electric field strength on the blade surface is enhanced with the increase of the rotation angle. Whereas when the blade rotates to a larger angle, the migration of charged particles under the influence of electric field and its own diffusion are comparatively sufficient, thus the concentration of charged particles near blade tip is decreased. This time the electric field strength on the blade surface is mainly subject to the influence of the electric field of thundercloud. With the increase of rotation angle, the electric field strength on the surface of blade tip is gradually reduced. 
In addition, the electric field strength of the blade tip increases with the increase of the rotating speed. When the rotating speed is $6 \mathrm{rpm}$ (revolutions per minute), the extremum of the electric field strength on the blade tip is $2.81 \mathrm{MV} / \mathrm{m}$, which is less than the corona initiation field strength on the blade tip. Therefore, it is difficult to initiate corona discharge. As for the rotating speed exceeding $9 \mathrm{rpm}$, the extremum of the electric field strength at the blade tip is greater than the corona onset field strength. Compared with the lowest speed (6 rpm), the extremum of electric field strength on the blade tip at the highest speed $(20 \mathrm{rpm})$ is $3.87 \mathrm{MV} / \mathrm{m}$, with an increase of $38 \%$. It is because as the rotating speed increases, the concentration of space charge near the blade tip is decreased, and the shielding effect of the electric field strength on the blade tip is consequently weakened.

\section{B. Quantitative analysis of the rotation impact on corona inception}

To describe the effect of blade rotating speed on the spatial distribution of charged particles, the definition of charge density descent vector is proposed, which is calculated from equation (7):

$$
\boldsymbol{l}_{\mathrm{c}}=-e \cdot \nabla\left(n_{+}+N_{+}\right)
$$

where, $e$ is the elementary charge.

The direction is along the steepest descent of charge density in the contour of charged particles distribution near the blade tip, as shown in Fig. 16. The value of charge density descent vector characterizes the reduction rate of charge density, and the larger the value of $\boldsymbol{l}_{\mathrm{c}}$ indicates that the faster the reduction rate of charge density near the blade tip. Thus, the space charge is reduced so that the weakening effect on the electric field strength near the blade tip is decreased.

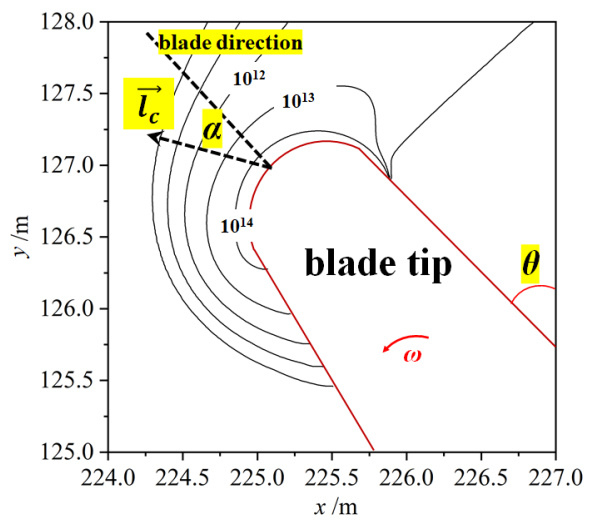

Fig. 16. Definition of charge density descent vector

In Fig. 16, the angle $\alpha$ between $\boldsymbol{l}_{\mathrm{c}}$ and the blade extension line is defined as the charge density offset angle, which characterizes the influence of blade rotation on the charged particles distribution near the blade tip. The smaller the angle $\alpha$ indicates that the more obvious the dragging effect of the blade rotation on charged particles distribution near the blade tip. And the distribution of charged particles along the direction of the extension line of the blade tip is decreased, while the distribution along the tangent line of the opposite direction of the blade rotation is expected to increase.
The values of $\left|\boldsymbol{l}_{\mathrm{c}}\right|$ and $\alpha$ when blade rotating to $30^{\circ}$ under different rotating speeds were calculated, as shown in Fig. 17.

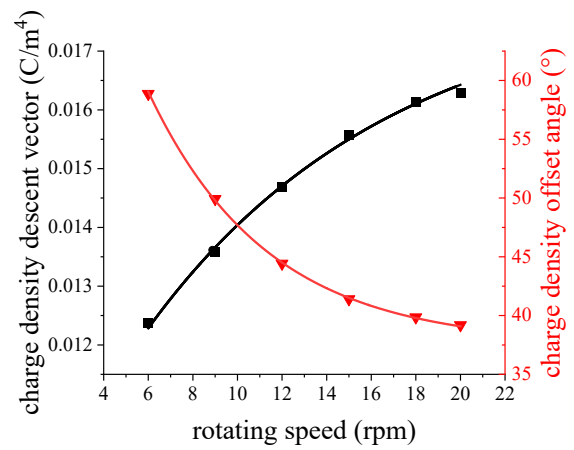

Fig. 17. Charge density descent vector and offset angle of charge density

From Fig. 17, it can be concluded that the charge density offset angle decreases with the increase of rotating speed. It indicates that as the rotating speed increases, the dragging effect of the blade on the charged particle distribution near the blade tip is more obvious, and the charged particles are distributed in a region further to the blade in the opposite direction of rotation. Therefore, a decreasing amount of space charge will accumulate near the tip of the blade. On the other hand, there is a tendency for $\left|\boldsymbol{l}_{\mathrm{c}}\right|$ to increase with the increase of the rotating speed. It indicates that the charged particles density near the blade tip decreases faster, and the amount of space charge decreases, resulting in a weakening effect on the electric field. Therefore, the electric field strength of the blade tip increases with the rise of the rotating speed.

The relation between $\left|\boldsymbol{l}_{\mathrm{c}}\right|$ and $n_{\mathrm{R}}, \alpha$ and $n_{\mathrm{R}}$ were obtained by exponential function fitting as shown in equation (8)

$$
\left\{\begin{array}{l}
\left|\boldsymbol{l}_{c}\right|=0.018-0.01 \times e^{-\frac{n_{R}}{10.98}} \\
\alpha=37.53+66.33 \times e^{-\frac{n_{R}}{5.33}}
\end{array}\right.
$$

The function shown in Eq. (7) were plotted in Fig. 17 (shown by the solid line), which was in good agreement with the calculated results, and the coefficient of correlation $\mathrm{R}$ of the fitting was more than 99\%. Combined with Fig. 17, it can be analyzed by equation (8) that as the blade speed increases, $\left|\boldsymbol{I}_{\mathrm{c}}\right|$ and $\alpha$ both tend to stabilize at value of $0.018 \mathrm{C} / \mathrm{m}^{4}$ and $37.53^{\circ}$, respectively, indicating that there is a limit to the effect of rotating speed on the space charge distribution near the blade tip. The linear velocity of the blade tip is already comparable to the mobility velocity of charged particles in the electric field environment when the blade speed is larger. With the further increase of rotating speed, the distribution of charged particles near the blade tip will become stable.

From the analysis in section IV(A), it is known that the rotating speed and the rotation angle of the blade together affect the electric field strength at the blade tip, and thus have an impact on the corona discharge inception. As shown in Fig. 18, a contour plot of the electric field strength on the blade tip surface of the wind turbine blade at different rotating speeds and rotation angles is calculated and used to determine the corona inception on the blade tip of the rotating wind turbine. 


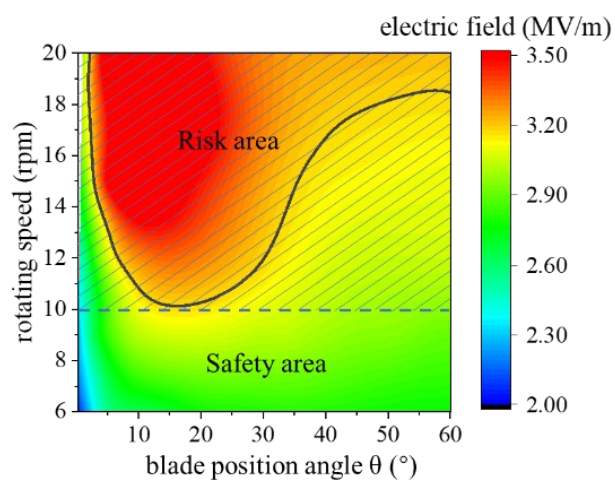

Fig. 18. Joint influence of rotating speed and rotating angle on the electric field on the blade tip

In Fig. 18, the solid line, which is found like U-shaped, shows the critical field strength of the corona initiation at the blade tip, i.e., $3.16 \mathrm{MV} / \mathrm{m}$. The area inside the curve refers to the field strength of the corona initiation, which satisfies the corona onset condition. As for the state of the blade outside the curve, the blade tip does not meet the field strength of corona initiation, thus it is not easy to develop into streamer and even leader development. The critical line is shown as U-shaped, which is caused that at different rotating speeds, the blade tip may reach the field strength of corona onset within different angle ranges rather than a certain rotation angle. The angle range at which the blade tip reaches the corona field strength is larger when the speed is higher, and when the speed exceeds 18 $\mathrm{rpm}$, the blade tip reaches the field strength of corona onset at almost any rotation angle. When the blade comes to a lower speed, the angle range of the blade tip to reach the corona field strength becomes smaller. For the wind turbine model established in this paper, when the rotating speed comes to 10 rpm, the blade tip reaches the corona field strength at $15^{\circ}$, reaching the minimum value of the U-shaped curve. And when the speed is lower than 10rpm, the blade tip does not reach the corona field strength at any blade position angle.

The dashed line in the Fig. 18 defines the dangerous rotating speed area (the shadow area) and the safe rotating speed area (the corresponding rotating speeds under U-shaped critical curve) of corona inception. It is to be noted that the rotating speed corresponding to the dashed line, which was defined as critical rotating speed, varies under different thundercloud field strengths.

Fig. 19 depicts the relation between critical rotating speed and the electric field strength of thundercloud from the calculation of the proposed model, which is approximately shown as linear variations fitted by:

$$
n_{\text {safe }}=13.94+0.10 \times E_{\text {cloud }}
$$

where $n_{\text {safe }}$ is critical rotating speed of wind turbine, $E_{\text {cloud }}$ represents to the field strength of negative thundercloud, varying from $-10 \mathrm{kV} / \mathrm{m}$ to $-60 \mathrm{kV} / \mathrm{m}$ [18], [21].

Depending on the climate and air quality where the wind turbine is located, the initial value of aerosol particles density $N_{0}$ is also different and can vary in the range of $5 \times 10^{9}$ to $2 \times$ $10^{11}\left(1 / \mathrm{m}^{3}\right)$ [22]. Since the density of neutral aerosol particles is around several orders of magnitude smaller than that of positive ions near the blade tip under thunderclouds condition, which has a limited effect on the electric field near the rotating wind turbine, and thus its effect on $n_{\text {safe }}$ is neglected. However, atmospheric observations showed that the ion mobility has a large variation above $2 \mathrm{~km}$ in altitude [23], which will consequently influence the space charge distribution and

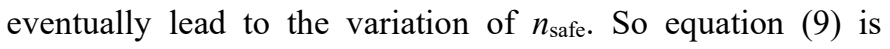
applicable to altitudes below $2 \mathrm{~km}$.

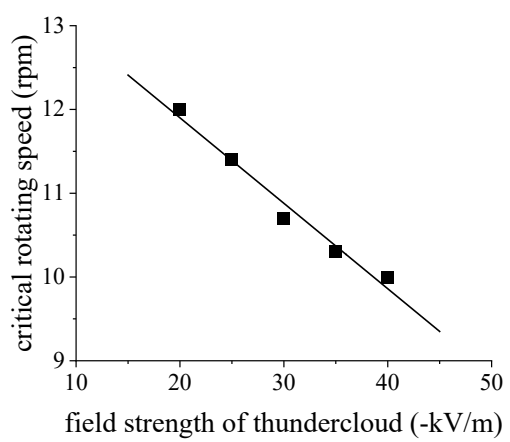

Fig. 19. Critical rotating speed under different thundercloud field strength

From equation (9), when the thundercloud electric field is $-139.4 \mathrm{kV}$, the critical rotating speed decreases to 0 . It shows that when the field strength of thunderclouds is greater than $-139.4 \mathrm{kV}$, the wind turbine should be shut down to avoid suffering the risk of lightning strikes. And when the field strength of thunderclouds is equal to 0 , that is, in sunny weather, the critical rotating speed is $13.94 \mathrm{rpm}$, which is in the range of working speed of wind turbine (6-20rpm). In fact, at this time, turbines are not affected by the proposed critical rotating speed and the actual speed should be regulated according to the wind farm. The critical rotating speed $n_{\text {safe }}$ is about 8 rpm when the thundercloud electric field reaches $-60 \mathrm{kV}$. Since the field strength of thunderclouds is measured mainly in the range of $-10 \mathrm{kV} / \mathrm{m}$ to $-60 \mathrm{kV} / \mathrm{m}$, in wind farms, it is recommended to reduce the rotating speed of the wind turbine below 8rpm in thundercloud conditions.

\section{CONCLUSIONS}

To study the impact mechanism of wind turbine rotation on the corona inception, a 2-D convection and diffusion model with dynamic meshing of charged particles in the neighboring space of a large-scale rotating wind turbine under thundercloud was established in this paper. The distribution characteristics of the charged ion flow in the neighboring domain of the blade tip and the impact mechanism of wind turbine rotation on the corona discharge have been analyzed, and the conclusions obtained are as follows.

(1) The distribution characteristic of charged particles in the neighboring domain of rotating wind turbine and its impact on field strength were studied. The rotation of the blade leads to a stripe-like distribution of charged particles, with a higher density of space charge distribution near the blade tip along the vertical direction and the rotating direction, which has a greater distortion effect on the electric field. The field strength on the blade tip is increasing with the growth of rotating speed and the 
extremum of electric field on the blade tip increased by $38 \%$ when the blade speed rises from 6rpm to 20rpm, which shows that as the rotating speed increases, the corona discharge is more likely to initiate at the blade tip.

(2) The impact mechanism of wind turbine rotation on the corona inception at the blade tip was studied, and the critical rotating speeds corresponding to corona inception probability were calculated under different thunderclouds-determined field strengths, based on which a safe boundary was defined. From the analysis of the combined effect of rotating speed and rotation angle on corona inception, $U$-shaped critical curve was proposed to determine the corona inception of rotating wind turbine. The minimum of the U-shaped line was defined as critical rotating speed, which is mainly influenced by the field strength of thundercloud. From the relationship between critical rotating speed of thundercloud field strength, it is recommended that the wind turbine is operated at a reduced speed below $8 \mathrm{rpm}$ under thundercloud conditions.

\section{REFERENCES}

[1] A. C. Garolera, S. F. Madsen, M. Nissim, J. D. Myers, and J. Holboell, "Lightning Damage to Wind Turbine Blades From Wind Farms in the U.S.," IEEE Transactions on Power Delivery, vol. 31, no. 3, pp. 1043-1049, Jun. 2016, DOI: 10.1109/TPWRD.2014.2370682.

[2] W. Chen et al., "Review of Research Progress in Lightning Damage and Protection of Wind Turbine Blades," High Voltage Engineering (in Chinese), vol. 45, no. 09, pp. 2782-2796, 2019, DOI: 10.13336/j.1003-6520.hve.20190831012.

[3] P. Christensen, J. L. Paulsen, M. L. Thøgersen, and T. Krogh, "Pålidelighedsmodel for havvindmølleparker," Riscopyright National Laboratory, Riso, vol. RisøR1346(DA), 2002, Accessed: Oct. 06, 2020. [Online]. Available: https://core.ac.uk/reader/13772070

[4] "IEC 61400-24: 2002 Wind turbine generator systems-Part 24: Lightning protection," IEC, 2002.

[5] "IEC 61400-24: 2010 Wind turbine generator systems-Part 24: Lightning protection," IEC, 2010.

[6] M. Miki, T. Miki, A. Wada, A. Asakawa, Y. Asuka, and N. Honjo, "Observation of lightning flashes to wind turbines," in 2010 30th International Conference on Lightning Protection (ICLP), Sep. 2010, pp. 1-7. DOI: 10.1109/ICLP.2010.7845869.

[7] J. Montanyà, O. van der Velde, and E. R. Williams, "Lightning discharges produced by wind turbines," Journal of Geophysical Research: Atmospheres, vol. 119, no. 3, pp. 1455-1462, 2014, DOI: 10.1002/2013JD020225.

[8] M. Ishii, M. Saito, D. Natsuno, and A. Sugita, "Lightning incidence on wind turbines in winter," in 2014 International Conference on Lightning Protection (ICLP), Oct. 2014, pp. 1734-1738. DOI: 10.1109/ICLP.2014.6973409.

[9] B. M. Radičević, M. S. Savić, S. F. Madsen, and I. Badea, "Impact of wind turbine blade rotation on the lightning strike incidence - A theoretical and experimental study using a reduced-size model," Energy, vol. 45, no. 1, pp. 644-654, Sep. 2012, DOI: 10.1016/j.energy.2012.07.032.

[10] Y. Wang, L. Qu, T. Su, Y. Nu, J. Xu, and X. Wen, "Experimental study of rotating wind turbine breakdown characteristics in large scale air gaps," Plasma Sci. Technol., vol. 19, no. 6, p. 064016, Apr. 2017, DOI: 10.1088/2058-6272/aa6743.

[11] M. A. Alonso and D. C. Irastorza, "Dynamic wind turbine lightning protection behaviour under storm conditions," in Proceedings of the 29th International Conference on Lightning Protection (ICLP2008), Uppsala, Sweden, 2008, pp. 9a-7-1-9a-7-6.

[12] L. Qu et al., "Simulation Study on Positive Corona Discharge of Receptors on Rotating Wind Turbine Blade Tips under Thundercloud Electric Fields," Energies, vol. 12, no. 24, Art. no. 24, Jan. 2019, DOI: 10.3390/en12244696.

[13] M. Becerra, V. Cooray, S. Soula, and S. Chauzy, "Effect of the space charge layer created by corona at ground level on the inception of upward lightning leaders from tall towers," Journal of Geophysical Research: Atmospheres, vol. 112, no. D12, 2007, DOI: 10.1029/2006JD008308.

[14] M. Becerra, "Glow corona generation and streamer inception at the tip of grounded objects during thunderstorms: revisited," J. Phys. D: Appl. Phys., vol. 46, no. 13, p. 135205, Feb. 2013, DOI: 10.1088/0022-3727/46/13/135205.

[15] G. Huang et al., "Improved 3-D Upwind FEM for Solving Ionized Field of HVDC Transmission Lines" Proceedings of the CSEE, vol. 33, no. 33, pp. 152-159+19, 2013, DOI:10.13334/j.0258-8013.pcsee.2013.33.019

[16] F. Xiao, B. Zhang, J. Mo, and J. He, "Calculation of 3-D Ion-Flow Field at the Crossing of HVdc Transmission Lines by Method of Characteristics," IEEE Transactions on Power Delivery, vol. 33, no. 4, pp. 1611-1619, Aug. 2018, DOI: 10.1109/TPWRD.2017.2737006.

[17] T. Lu, H. Feng, Z. Zhao, and X. Cui, "Analysis of the Electric Field and Ion Current Density Under Ultra High-Voltage Direct-Current Transmission Lines Based on Finite Element Method," IEEE Transactions on Magnetics, vol. 43, no. 4, pp. 1221-1224, Apr. 2007, DOI: 10.1109/TMAG.2006.890960.

[18] X. Qie, S. Soula, and S. Chauzy, "Influence of ion attachment on the vertical distribution of the electric field and charge density below a thunderstorm," Annales Geophysicae, vol. 12, no. 12, pp. 1218-1228, Dec. 1994, DOI: 10.1007/s00585-994-1218-6.

[19] F. Alessandro and G. Berger, "Laboratory studies of corona emissions from air terminals," J. Phys. D: Appl. Phys., vol. 32, no. 21, pp. 2785-2790, Oct. 1999, DOI: 10.1088/0022-3727/32/21/311.

[20] F. W. Peek, Dielectric phenomena in high voltage engineering. New York: McGraw-Hill Book Company, 1929.

[21] H. Zhang et al., "The charge structure in a thunderstorm based on three-dimensional electric field sonde," Chinese Journal of Geophysics (in Chinese), vol. 64, no. 04, pp. 1155-1166, 2021, DOI: $10.6038 /$ cjg202100187.

[22] S. Twomey, Atmospheric aerosols. New York: Elsevier Scientific Publishing Co., 1977.

[23] R. E. Meyerott, J. B. Reagan, and R. G. Joiner, "The mobility and concentration of ions and the ionic conductivity in the lower stratosphere," Journal of Geophysical Research: Space Physics, vol. 85, no. A3, pp. 1273-1278, 1980, DOI: 10.1029/JA085iA03p01273.

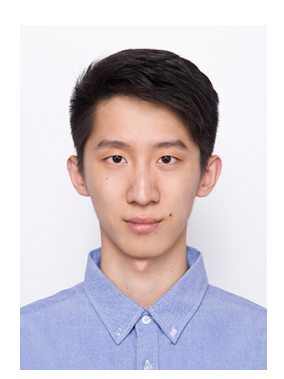

Wanshui Yu was born in Henan, China in 1995. He received the B.Sc. degree in Electrical Engineering from Zhengzhou University in 2017. He is pursuing a doctor degree major in electrical engineering at the School of Electrical and Electronic Engineering, North China Electric Power University, China. His interests mainly include long gap discharges and lightning protection on

wind turbine.

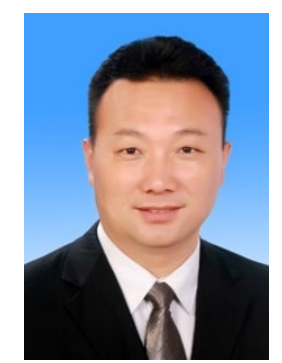

Qingmin Li (M'07) received the B.Sc., M.Sc., and Ph.D. degrees in electrical engineering from Tsinghua University, Beijing, China, in 1991, 1994, and 1999, respectively.

In 1996, he joined Tsinghua University as a Lecturer. In 2000, he joined the University of Liverpool, Liverpool, U.K., and later at the University of Strathclyde, Glasgow, U.K., as a Post-Doctoral Research Fellow. From 2003 to 2011, he was a Professor of electrical engineering with Shandong University, Jinan, China. He is currently a Professor of electrical engineering with North China Electric Power University, Beijing. His current research interests include high voltage engineering, applied electromagnetics and condition monitoring and fault diagnostics. 
Dr. Li is a member of IET.

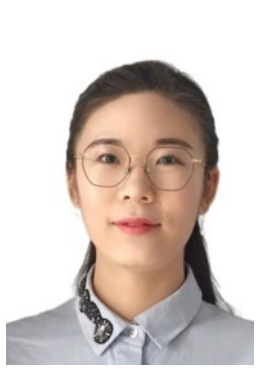

Jiyao Zhao was born in Hebei, China in 1996. She received the B.Sc. degree in Electrical Engineering from Beijing University of Civil Engineering and Architecture in 2019. She is pursuing a master degree major in electrical engineering at the School of Electrical and Electronic Engineering, North China Electric Power University, China. Her interests mainly include offshore wind turbines and space charge distribution characteristics.

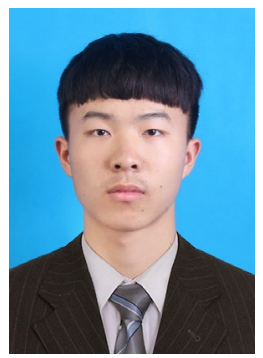

Hongbo Li received the B.Sc. degree in electrical engineering from Changsha University of Science \& Technology, Changsha, China, in 2018, and received the M.Sc. degree in electrical engineering from North China Electric Power University, Beijing, China, in 2021. His research interests include fault detection and location of lightning protection system on

wind turbine.

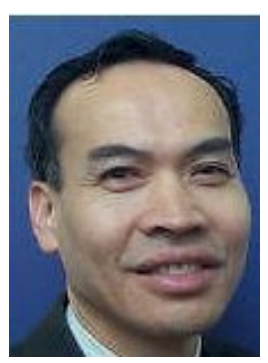

Wah Hoon Siew (M'95) received the B.Sc. degree (Hons.) in electronic and electrical engineering, the M.B.A. degree, and the Ph.D. degree in electronic and electrical engineering from the University of Strathclyde, Glasgow, U.K. He is currently a Professor with the Department of Electronic and Electrical Engineering, University of Strathclyde. His current research interests include large system electromagnetic compatibility, cable diagnostics, lightning protection, and wireless sensing systems. Dr. Siew is a Convener of the CIGRE WG C4.208 and a member of the Technical Advisory Panel for the IET Professional. 